\title{
Metales pesados en macromicetos del manglar de la bahía Cispatá, Córdoba, Colombia
}

\section{Heavy metals in macromycetes of the mangrove of the Cispatá bay, Córdoba, Colombia}

\author{
Edith-de-Jesús Cadavid-Velásquez ; Nabi-del-Socorro Pérez-Vásquez ${ }^{2}$; José Marrugo-Negrete ${ }^{3}$
}

\begin{abstract}
'Licenciada en Biología y Química, M.Sc. Universidad de Córdoba. Montería, Córdoba, Colombia; e-mail: edithcadavid@correo.unicordoba.edu.co; (Dhttps://orcid.org/0000-0003-0115-8857

Licenciada en Biología y Química, M.Sc. Estudiante de Doctorado. Universidad de Córdoba, Grupo de Investigación Biodiversidad. Montería, Córdoba, Colombia. e-mail: ndperezvasquez@correo.unicordoba.edu.co; Dhttps://orcid.org/0000-0002-5349-0598
\end{abstract}

${ }^{3}$ Ing. Químico, Ph.D. Universidad de Córdoba, Grupo de Investigación de Toxicología y Gestión Ambiental. Montería, Córdoba, Colombia. e-mail: jmarrugo@ correo.unicordoba.edu.co; Dhttps://orcid.org/0000-0002-3181-7529

Cómo citar: Cadavid-Velásquez, E. de J.; Pérez-Vásquez, N. del S.; Marrugo-Negrete, J. 2019. Metales pesados en macromicetos del manglar de la bahía Cispatá, Córdoba, Colombia. Rev. U.D.C.A Act. \& Div. Cient. 22(2):e1082. https://doi.org/10.31910/rudca.v22. n1.2019.1082

Artículo de acceso abierto publicado por Revista U.D.C.A Actualidad \& Divulgación Científica, bajo una licencia Creative Commons CC BY-NC 4.0

Recibido: Noviembre 20 de 2018

Aceptado: Junio 7 de 2019

Editado por: Ingeborg Zenner de Polanía

\section{RESUMEN}

Desde el 2008, se evidenció la presencia de metales pesados en la zona estuarina de la Bahía de Cispatá, en agua, en sedimento, en peces y en aves, de ahí, el interés de evaluarlo en los macromicetos del manglar, que tienen una función degradadora de la materia orgánica, contribuyendo a mantener el equilibrio dinámico de la naturaleza, absorbiendo, incluso, sustancias tóxicas y elementos esenciales en altas concentraciones. Este estudio determinó las concentraciones de 9 metales pesados ( $\mathrm{Ni}, \mathrm{Cu}, \mathrm{Mn}, \mathrm{Cr}, \mathrm{Zn}, \mathrm{Co}, \mathrm{Hg}, \mathrm{Pb}$ y $\mathrm{Cd}$ ) en 19 géneros de hongos macromicetos, asociados al manglar de la bahía Cispatá, recolectados en tres sitios de muestreo, en tres períodos de lluvias, durante el 2016 y 2017. Los macromicetos, se secaron, pulverizaron y después de su digestión completa, en una mezcla de ácido nítrico y ácido clorhídrico, se cuantificaron los metales pesados. Los resultados indicaron que todos los hongos concentraron metales pesados. Para las concentraciones de metales pesados no se encontraron diferencias estadísticamente significativas entre macromicetos jóvenes y adultos $(\mathrm{p}=0,926)$; sin embargo, se observaron correlaciones estadísticamente significativas entre Co$\mathrm{Ni}, \mathrm{Hg}-\mathrm{Zn}, \mathrm{Pb}-\mathrm{Mn}, \mathrm{Pb}-\mathrm{Ni}, \mathrm{Pb}-\mathrm{Co}, \mathrm{Cd}-\mathrm{Mn}, \mathrm{Cd}-\mathrm{Co}, \mathrm{Cd}-\mathrm{Hg}$ y Cd-Ni. Los macromicetos han sido poco estudiados en las zonas costeras colombianas y aunque en este estudio las concentraciones de los metales pesados no son alarmantes, los macromicetos reflejan el estatus de estos contaminantes en la zona.

Palabras clave: concentración; contaminación; estuario; macrohongos (CAB Thesaurus).

\section{ABSTRACT}

Since 2008, the presence of heavy metals in the estuarine zone of the Bay of Cispatá was evidenced in water, sediment, fish and birds; hence the interest to evaluate it in the mangrove macromycetes, which have a degrading function of organic matter, helping to maintain the dynamic balance of nature by absorbing even toxic 
substances and essential elements in high concentrations. This study determined the concentrations of 9 heavy metals $(\mathrm{Ni}, \mathrm{Cu}$, $\mathrm{Mn}, \mathrm{Cr}, \mathrm{Zn}, \mathrm{Co}, \mathrm{Hg}, \mathrm{Pb}$ and $\mathrm{Cd}$ ) in 19 genera of macromycetes fungi associated with the mangrove of Cispatá Bay, collected in three sampling sites in three rainy periods during 2016 and 2017. The macromycetes were dried, pulverized and after their complete digestion in a mixture of nitric acid and hydrochloric acid the heavy metals were quantified. The results indicated that all fungi concentrated heavy metals. For the concentrations of heavy metals, no statistically significant differences were found between young and adult macromycetes $(p=0.926)$, however, statistically significant correlations were observed between $\mathrm{Co}-\mathrm{Ni}$, $\mathrm{Hg}-\mathrm{Zn}, \mathrm{Pb}-\mathrm{Mn}, \mathrm{Pb}-\mathrm{Ni}$, $\mathrm{Pb}-\mathrm{Co}, \mathrm{Cd}-\mathrm{Mn}, \mathrm{Cd}-\mathrm{Co}, \mathrm{Cd}-\mathrm{Hg}$ and $\mathrm{Cd}-\mathrm{Ni}$. Macromycetes have been little studied in Colombian coastal areas, and although in this study the concentrations of heavy metals are not alarming, macromycetes reflect the status of these pollutants in the area.

Keywords: concentration; pollution; estuary; macrohongos (CAB Thesaurus).

\section{INTRODUCCIÓN}

La importancia de los macromicetos radica en su papel dentro del ciclo del carbono, degradando materiales, como la lignina; por tal motivo es frecuente colectarlos, asociados a árboles en descomposición, que pueden degradar una gran cantidad de compuestos de estructura aromática, ya que estas enzimas son inespecíficas y son excretadas por el hongo ante la presencia del sustrato, como única fuente de carbono (Leonowicz et al. 1999; Lodge et al. 2004; Ortiz-Moreno, 2010; Chan-Cupul et al. 2016). La alta productividad de materia orgánica asociada a la degradación de biomasa vegetal procedente de los árboles y de la vegetación en el manglar, favorece el crecimiento y el desarrollo de hongos macromicetos, estableciendo que pueden habitar varios nichos distintos en dichos ecosistemas (Sosa-Rodríguez et al. 2009).

Los macromicetos han demostrado presentar una alta capacidad para degradar compuestos xenobióticos (Rivero et al. 2016). Algunos metales pesados son esenciales en los procesos fisiológicos normales; sin embargo, en altas concentraciones tienen efectos nocivos sobre la salud humana y la biota al ingresar a las cadenas tróficas (LorenzoMárquez et al. 2015). Los organismos son capaces de proveer información de los cambios ambientales provocados por el hombre (Argota et al. 2013).

Estudios han demostrado la presencia de metales pesados en la bahía Cispatá, como cadmio (Cd), cobalto (Co), cobre $(\mathrm{Cu})$, cromo $(\mathrm{Cr})$, manganeso $(\mathrm{Mn})$, mercurio $(\mathrm{Hg})$, níquel $(\mathrm{Ni})$, plomo $(\mathrm{Pb})$ y aluminio (Al) en agua, en sedimento, en peces y en aves (Burgos et al. 2014; Burgos-Núñez et al. 2017). Estos metales, se encuentran en la bahía, por la operación del distrito de riego de la Doctrina y del puerto de embarque de petróleo, así como a la ganadería extensiva, la construcción y posterior abandono de estanques para cría de camarones, la disposición inadecuada de residuos domésticos y el desarrollo de actividades turísticas no planificadas, contribuyendo a la llegada de estos elementos al estuario (Feria et al.
2010; Arteaga et al. 2017). Se encontraron concentraciones de Cu, $\mathrm{Ni}, \mathrm{Pb}, \mathrm{Cd}, \mathrm{Hg}$ y Zn en suelos, en un área circundante a la bahía, por Marrugo et al. (2017), lo que sugiere que la contaminación se deriva, principalmente, de las prácticas agrícolas, metales que fácilmente pueden llegar por escorrentías a la zona estuarina; de hecho, varios fertilizantes que contienen fracciones pequeñas de $\mathrm{Zn}$ y $\mathrm{Cu}$, se utilizan para incorporar estos elementos en suelos deficientes, mientras que el arsénico (As), $\mathrm{Cd}$ y $\mathrm{Hg}$ son componentes de algunos fungicidas. Además, el $\mathrm{Cu}$ se emplea como alguicida, mientras que el $\mathrm{Cd}$ y $\mathrm{Zn}$ pueden estar presente en los fertilizantes de fosfato (Burgos-Núñez et al. 2017). El objetivo de este estudio fue determinar las concentraciones de metales pesados $\mathrm{Ni}, \mathrm{Cu}, \mathrm{Mn}$, $\mathrm{Cr}, \mathrm{Zn}, \mathrm{Co}, \mathrm{Hg}, \mathrm{Pb}$ y $\mathrm{Cd}$ en hongos macromicetos, colectados en la Bahía Cispatá y reflejar, a través de organismos, el nivel de estos contaminantes en la zona.

\section{MATERIALES Y MÉTODOS}

Área de estudio. La bahía de Cispatá está ubicada en la costa Caribe colombiana, al norte del departamento de Córdoba, en el extremo suroeste del golfo de Morrosquillo, entre latitudes $09^{\circ} 25^{\prime} 12$ '$09^{\circ} 20^{\prime} 8^{\prime \prime} \mathrm{N}$ y longitudes $75^{\circ} 47^{\prime} 37^{\prime \prime}-75^{\circ} 55^{\prime} 30^{\prime \prime} \mathrm{W}$. La zona está influenciada por las corrientes marinas y desbordes de aguas del río Sinú, que conformaron las llanuras litoral y deltaica actuales, que han originado los manglares, las ciénagas, las marismas y los caños, en el antiguo delta del río (Dueñas et al. 2012).

Recolección e identificación de macromicetos. Los macromicetos fueron recolectados en la bahía Cispatá (Figura 1), durante el periodo de lluvias, en tres salidas de campo, entre 2016 y 2017, utilizando el método oportunístico (Mata et al. 2003). Para la toma de muestra, se extrajo el carpóforo (cuerpo fructífero), con la ayuda de una espátula, que se enterró tres centímetros hacia abajo de la base e introduciéndola en una bolsa de papel Kraft (Mata et al. 2009); se transportó hasta el laboratorio, donde se efectuó observación morfológica de los ejemplares, mediante un estereoscopio Nikon SMZ-2T, donde se identificó hasta la categoría de género, mediante claves taxonómicas (Franco \& Uribe, 2000; Mata et al. 2003; Mata et al. 2009) y su estado de desarrollo, apoyado en literatura especializada. Se midió el tamaño del carpóforo (indicador de edad), desde el estado juvenil (primordio), hasta el adulto (himenio), además del color (Alonso et al. 2004).

Determinación de metales pesados. Se tomaron de 50 a $100 \mathrm{~g}$ del cuerpo fructífero de los géneros colectados. Las muestras fúngicas libres de desechos forestales y de sedimento, se secaron en una estufa a $60^{\circ} \mathrm{C}$, hasta obtener un peso constante (Moyano et al. 2010). Posteriormente, se molieron en mortero de ágata y los materiales resultantes, se codificaron, almacenándose en recipientes de plástico herméticos, para su análisis; las concentraciones de Mn, $\mathrm{Cu}, \mathrm{Cr}, \mathrm{Ni}, \mathrm{Zn}$ y Co, se determinaron por espectrofotometría de absorción atómica, con llama de aire-acetileno (FAAS); el Pb y Cd, por espectrofotometría de Absorción atómica, por horno de grafito (GFAAS), S Series, modelo S4, marca Thermo Electron Corporation 3015A (EPA) y el Hg, se determinó mediante un analizador directo de mercurio DMA 80, de acuerdo con el método EPA 7473. Se tomó 


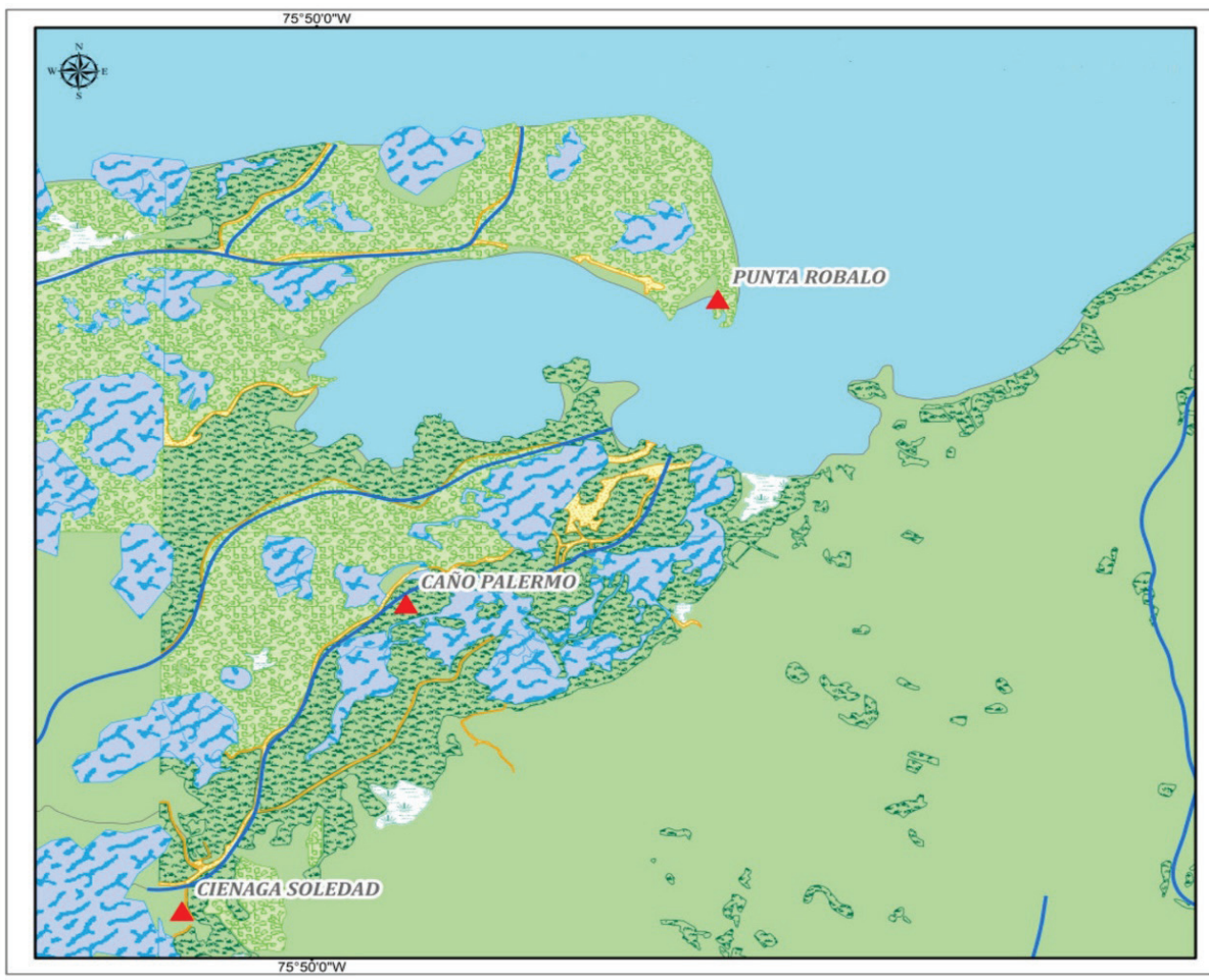

Figura 1. Sitios de muestreo durante las tres salidas. Bahía Cispatá, Córdoba-Colombia.

una muestra de un gramo (peso seco) y se adicionaron $10 \mathrm{ml}$ de una mezcla de ácido nítrico y ácido clorhídrico, en relación de 6:2, durante dos horas o hasta su digestión completa (Macías \& Calvo de Anta, 2008). Las muestras, se llevaron al laboratorio para su análisis de metales pesados (Madero \& Marrugo, 2011; Marrugo et al. 2017).

Análisis estadístico. Los datos de las concentraciones de metales pesados en géneros de hongos macromicetos, se expresan con la media de las concentraciones en peso seco de los análisis por triplicado \pm la desviación estándar. Se aplicaron diagramas de boxplot, para verificar la existencia de datos atípicos. Posteriormente, las pruebas de normalidad Anderson-Darling, Jarque-Bera, para verificar la distribución paramétrica de los datos, aplicándose la prueba de Kruskal-Wallis, para establecer diferencias significativas. Con el coeficiente de correlación de Spearman, se determinaron las relaciones entre metales pesados presentes en los hongos. Se aplicó la prueba de independencia (chi cuadrado), para el nivel de significancia en cada metal, según la etapa de desarrollo de los hongos. Para determinar agrupaciones y asociaciones de comportamientos de géneros de hongos o entre metales pesados, se emplearon dendrogramas. El criterio de significancia para los análisis estadísticos, se estableció en $\mathrm{p}<0,05$ (Miller \& Miller, 2002). El tratamiento de los resultados fue realizado mediante el estadístico $\mathrm{R}$ 3.5.1.

\section{RESULTADOS Y DISCUSIÓN}

Se obtuvieron 19 géneros de hongos macromicetos de 79 muestras, recolectadas con la siguiente frecuencia total, durante las 3 salidas, de los tres sitios de muestreo: Trametes (15), Cerrena (11), Crepidotus (10), Daldinia (6), Pleorotus (5), Polyporus (4), Marasmiellus (4), Postia (4), Earliella (3), Perenniporia (3), Daedalea (2), Lenzites (2), Tyromices (2), Hexagonia (2), Auricularia (1), Ceriporia (1), Chandrosterum (1), Mycena (1) y Oudemansiella (1).

El género de mayor frecuencia recolectada fue Trametes (15 individuos), seguido de Cerrena (11 individuos) y Crepidotus (10 individuos); asimismo, en el trabajo de González et al. (2005), Trametes fue el género más abundante y aducen que son géneros cosmopolitas saprófitos o parásitos de árboles, con abundantes registros en las zonas tropicales. Los hongos, tanto por su capacidad hidrolítica como por su distribución, son organismos lignocelulósicos por excelencia; entre ellos, existen algunos con mayor capacidad degradativa de lignina (Joseleau \& Ruel, 1994; Carlile et al. 2001), entre los que se incluyen los anteriores géneros. De igual modo, los géneros Auricularia, Ceriporia, Chandrosterum, Mycena y Oudemansiella presentaron una menor frecuencia de colecta de hongos macromicetos (1 individuo).

La estación de Caño Palermo registró menos individuos de géneros de hongos macromicetos (17), durante las 3 salidas y se puede atribuir a las diferencias en cuanto al grado de perturbación existente, ya que las estaciones Ciénaga soledad (37) y Punta robalo (25) son zonas de abundante vegetación (Sánchez et al. 2004).

La primera salida, se realizó en julio y fue en la que menor macromicetos se recolectaron (16), época de aguas en descenso. 
La segunda salida, época de aguas altas, en octubre, se colectaron 36 individuos, siendo la más abundante en hongos; la mayoría de géneros en este estudio son miembros de los órdenes Polyporales y Agaricales, que se adaptan particularmente bien a las condiciones cambiantes de temperatura, humedad relativa y pluviosidad, empleando estrategias de dispersión asociadas al viento y a la lluvia (Lodge et al. 2004). La tercera salida fue en mayo, época de aguas en ascenso y se recolectaron 27 individuos de macromicetos.

Se determinaron concentraciones de metales pesados en todos los hongos macromicetos recolectados, donde, estadísticamente, se establecieron valores de referencia $\left(\mathrm{n}^{\circ}\right.$ de frecuencia de recolecta, mínima concentración y máxima concentración de metales pesados, promedios de concentraciones y desviación estándar), para estimar la variación de cada metal pesado, en cada hongo recolectado. En la tabla 1, se observan los hongos macromicetos más concentradores, promedios totales de concentración de metales pesados de las tres estaciones, durante las tres salidas. Los hongos que presentaron mayores concentraciones de manganeso $(\mathrm{Mn})$ fueron Crepidotus, Polyporus y Mycena; de cromo (Cr), Cerrena, Crepidotus, Marasmiellus; niquel (Ni), Mycena, Hexagonia y Crepidotus; cobre $(\mathrm{Cu})$, Polyporus, Crepidotus y Mycena; zinc ( $\mathrm{Zn}$ ), Crepidotus, Mycena y Postia; cobalto (Co), Crepidotus, Trametes, Oudemansiella y Perenniporia; mercurio (Hg), Trametes, Pleorotus y Oudemansiella; plomo (Pb), Chandrosterum, Crepidotus y Postia y cadmio (Cd), Chandrosterum, Mycena y Oudemansiella.

Tabla 1. Promedios totales de concentración de metales pesados ( $\mathrm{mg} / \mathrm{kg}$ de peso seco) de las tres estaciones, durante las tres salidas en hongos macromicetos más concentradores, en la bahía de Cispatá, Colombia.

\begin{tabular}{|c|c|c|c|c|c|c|}
\hline MP & Género & $\mathbf{N}^{\circ}$ & Mín. & Máx. & Prom. & D. S. \\
\hline \multirow{3}{*}{ Mn } & Crepidotus & 10 & 1,11 & 171,26 & 37,37 & 58,01 \\
\hline & Mycena & 1 & 71,95 & 71,95 & 71,95 & 0,00 \\
\hline & Polyporus & 4 & 0,50 & 88,51 & 28,40 & 40,83 \\
\hline \multirow{3}{*}{$\mathrm{Cu}$} & Crepidotus & 10 & 0,59 & 21,45 & 4,24 & 6,22 \\
\hline & Mycena & 1 & 19,99 & 19,99 & 19,99 & 0,00 \\
\hline & Polyporus & 4 & 0,28 & 27,54 & 8,37 & 12,85 \\
\hline \multirow{3}{*}{$\mathrm{Hg}$} & Oudemansiella & 1 & 0,05 & 0,05 & 0,05 & 0,00 \\
\hline & Pleorotus & 5 & 0,006 & 0,05 & 0,03 & 0,02 \\
\hline & Trametes & 15 & 0,004 & 0,06 & 0,02 & 0,01 \\
\hline \multirow{3}{*}{$\mathrm{Ni}$} & Crepidotus & 10 & 0,06 & 9,72 & 4,26 & 3,90 \\
\hline & Hexagonia & 2 & 4,72 & 8,88 & 6,80 & 2,94 \\
\hline & Mycena & 1 & 11,59 & 11,59 & 11,59 & 0,00 \\
\hline \multirow{3}{*}{$\mathrm{Cd}$} & Chandrosterum & 1 & 0,107 & 0,107 & 0.107 & 0,00 \\
\hline & Mycena & 1 & 0,103 & 0,103 & 0.103 & 0,00 \\
\hline & Oudemansiella & 1 & 0,077 & 0,077 & 0,077 & 0.07 \\
\hline \multirow{3}{*}{$\mathrm{Cr}$} & Cerrena & 11 & 0,0001 & 8,11 & 2,32 & 2,35 \\
\hline & Crepidotus & 10 & 0,16 & 8,28 & 3,41 & 2,67 \\
\hline & Marasmellus & 4 & 0,0001 & 8,44 & 3,36 & 3,97 \\
\hline \multirow{3}{*}{$\mathrm{Zn}$} & Crepidotus & 10 & 4,04 & 36,78 & 14,64 & 10,25 \\
\hline & Mycena & 1 & 30,31 & 30,31 & 30,31 & 0,00 \\
\hline & Postia & 4 & 0,00 & 23,58 & 10,40 & 11,73 \\
\hline \multirow{3}{*}{$\mathrm{Pb}$} & Chandrosterum & 1 & 4,129 & 4,13 & 4,13 & 0,00 \\
\hline & Crepidotus & 10 & 0,007 & 2,89 & 0,48 & 0,90 \\
\hline & Postia & 4 & 0,0008 & 2,59 & 0,70 & 1,27 \\
\hline \multirow{3}{*}{ Co } & Crepidotus & 10 & 0,13 & 10,43 & 4,86 & 3,80 \\
\hline & Oudemansiella & 1 & 9,88 & 9,88 & 9,88 & 0,00 \\
\hline & Perenniporia & 3 & 0,0001 & 9,16 & 3,37 & 5,03 \\
\hline
\end{tabular}

$\mathrm{N}^{\circ}=$ Cantidad de muestras; $\mathrm{MP}=$ metal pesado; Prom. = Promedio; Mín. $/$ Máx.= Mínimos/Máximos; D.S.=Desviación estándar 
La prueba de Kruskal-Wallis con $\mathrm{p}=0,508$, teniendo en cuenta las tres salidas, indicó que solo el $\mathrm{Cr}$ no presenta influencia en las diferentes salidas, mientras que la prueba detecta diferencias de distribuciones del resto de metales pesados para las diferentes salidas. Para los tres sitios de muestreo, la prueba de Kruskal-Wallis $\mathrm{p}=0,001$ indica que los diferentes lugares afectan la distribución del $\mathrm{Cu}$, mientras que, en el resto de metales pesados, no hay cambio significativo.

De la misma manera, esta misma prueba Kruskal-Wallis, con $\mathrm{p}=0,926$, indicó que todos los metales tienen el mismo comportamiento, al concentrarse en todos los géneros colectados y, para cada uno de los metales, sí existió dependencia entre hongos jóvenes y adultos, cuyo menor valor fue para el Ni (Chi-cuadrado, $\mathrm{p}=0,368)$.

La correlación de metales pesados en hongos muestra relaciones directamente proporcionales y estadísticamente significativas (Spearman, p<0,05), entre Co-Ni, Hg-Zn, Pb-Mn, Pb-Ni, Pb-Co, Cd-Mn, Cd-Co, Cd-Hg y Cd-Ni, según muestra la tabla 2.
La concentración más altas en todos los macromicetos fue el manganeso (Mn) y el hongo que presentó la más alta concentración de este metal fue del género Crepidotus $(171,26 \mathrm{mg} / \mathrm{kg})$, resultados que se contrastan con el estudio de Durkan et al. (2010), en ecosistemas similares, de la cuenca de varios brazos del río Büyük Menderes, en la parte suroeste de Turquía, donde obtuvo concentraciones hasta $176,4 \mathrm{mg} / \mathrm{kg}$, en hongos del lugar, señalando, a su vez, que las altas concentraciones de este metal, se debieron a acciones antrópicas. De la misma manera, el Mn entra a los ecosistemas por acciones naturales, como por actividades humanas, que tienen incidencia sobre la concentración de este metal en las zonas acuíferas (Herrera et al. 2012). La mayor concentración de cobre, se observó en el género Polyporus $(27,54 \mathrm{mg} / \mathrm{kg})$ que, al contrastar estos resultados de nuevo con los reportados por Durkan et al. (2010), se encontraron concentraciones entre $9,654 \mathrm{mg} / \mathrm{kg}-39,55 \mathrm{mg} / \mathrm{kg}$, siendo similares con los de este estudio. La presencia de cobre en el sistema manglar de la Bahía Cispatá, se relaciona con el uso de pesticidas, herbicidas y abonos, por actividades agrícolas desarrolladas en la región (Burgos et al. 2014; Burgos-Núñez et al. 2017).

Tabla 2. Coeficientes de correlación de Spearman entre metales pesados en hongos macromicetos.

\begin{tabular}{|c|c|c|c|c|c|c|c|c|c|}
\hline Met.pesados & $\mathrm{Mn}$ & $\mathrm{Cr}$ & $\mathrm{Ni}$ & $\mathrm{Cu}$ & $\mathrm{Zn}$ & $\mathrm{Co}$ & $\mathrm{Hg}$ & $\mathrm{Pb}$ & $\mathrm{Cd}$ \\
\hline $\mathrm{Mn}$ & 1 & 0,8403 & 0.0722 & 0.2071 & 0.3259 & 0.0980 & 0.2738 & $0.0064^{*}$ & $0.0336^{*}$ \\
\hline $\mathrm{Cr}$ & 0,002 & 1 & 0.4980 & 0.6090 & 0.0699 & 0.8650 & 0.5269 & 0.1999 & 0.7623 \\
\hline $\mathrm{Ni}$ & 0,169 & 0,026 & 1 & 0.2323 & 0.8060 & $0.0002^{*}$ & 0.9548 & $0.0277^{*}$ & $<.0001^{*}$ \\
\hline $\mathrm{Cu}$ & 0,087 & 0,015 & 0,078 & 1 & 0.8601 & 0.2767 & 0.7479 & 0.7623 & 0.4426 \\
\hline $\mathrm{Zn}$ & 0,054 & 0,171 & 0,003 & 0,002 & 1 & 0.0733 & $0.0019^{*}$ & 0.8799 & 0.6633 \\
\hline $\mathrm{Co}$ & 0,145 & 0,002 & 0,554 & 0,065 & 0,167 & 1 & 0.2511 & $0.0042^{*}$ & $0.0009^{*}$ \\
\hline $\mathrm{Hg}$ & 0,066 & 0,023 & 0,000 & 0,006 & 0,424 & 0,072 & 1 & 0.6314 & 0.8551 \\
\hline $\mathrm{Pb}$ & 0,346 & 0,090 & 0,242 & 0,005 & 0,001 & 0,373 & 0,013 & 1 & $0.0089^{*}$ \\
\hline $\mathrm{Cd}$ & 0,227 & 0,005 & 0,784 & 0,033 & 0,011 & 0,466 & 0,002 & 0,323 & 1 \\
\hline
\end{tabular}

* Nivel de significancia $(\mathrm{p}>0,05)$

El género Marasmiellus presentó la máxima concentración de Cr $(8,44 \mathrm{mg} / \mathrm{kg})$, superior a los reportados por Demirbas (2001), con concentraciones entre $0,60 \mathrm{mg} / \mathrm{kg}-1,68 \mathrm{mg} / \mathrm{kg}$, en la región turca del Mar Negro, en Turquía y Mendil et al. (2005), entre 1,1mg/kg $4,4 \mathrm{mg} / \mathrm{kg}$, en la región de Ordu, Turquía, proveniente por contaminación de residuos domésticos; asimismo, en la Bahía Cispatá, este resultado da cuenta de contaminación proveniente actividad petrolera (Burgos-Núnez et al. 2017) y a una industria de curtiembre en la que se utilizaban sales de $\mathrm{Cr}$ en el pasado.

El macromiceto Mycena concentró níquel en una proporción de $11,59 \mathrm{mg} / \mathrm{kg}$, sobrepasando a los valores encontrados por Svoboda et al. (2000), en un área muy contaminada por la minería, en el este de Eslovaquia, por hongos del lugar, por la fundición de minerales polimetálicos y las emisiones de una fundición de mercurio y cobre (Durkan et al. 2010), donde las concentraciones variaron entre $0,4 \mathrm{mg} / \mathrm{kg}-2 \mathrm{mg} / \mathrm{kg}$. La presencia de este metal en la Bahía
Cispatá, se debe a actividades que se asocia con la actividad antrópica desarrollada en el distrito de riego de La Doctrina, por la aplicación de plaguicidas, donde se ha cambiado, de forma progresiva, el uso del suelo, pasando de actividades de agricultura intensiva, a desarrollar actividades piscícolas (Sánchez, 2010).

El género que más concentró Zn fue el género Crepidotus $(36,78 \mathrm{mg} / \mathrm{kg})$, mientras que, en otras investigaciones, se reportaron rangos de concentración entre $19,66 \mathrm{mg} / \mathrm{kg}-75,91 \mathrm{mg} / \mathrm{kg}$, por Durkan et al. (2010), en macromicetos del orden Agaricales, que incluye a los del género Crepidotus; así, en el río Mendere como en la Bahía Cispatá, la presencia de este elemento, se debe a la acción antrópica; gran aporte de este metal, se debe por la industria camaronera en épocas pasadas, en la zona estaruarina.

La mayor concentración de Co, se encontró en el Trametes $(9,99 \mathrm{mg} / \mathrm{kg})$, aunque es un valor relativamente alto, si los 
comparamos con los dados a conocer en otros tipos de géneros de macromicetos, por Borovička \& Řanda (2007), que fue entre $5,85 \mathrm{mg} / \mathrm{kg}$ y $0,04 \mathrm{mg} / \mathrm{kg}$, en regiones de las Repúblicas Checa y Eslovaca.

El macromiceto que más concentró $\mathrm{Hg}$ fue Oudemansiella $(0,055 \mathrm{mg} / \mathrm{kg})$, mientras que los valores reportados por Svoboda et al. (2000), fueron entre $1,45 \mathrm{mg} / \mathrm{kg}-2,86 \mathrm{mg} / \mathrm{kg}$ y los de Rzymski et al. (2016) oscilaron entre $44 \mathrm{mg} / \mathrm{kg}-53 \mathrm{mg} / \mathrm{kg}$, recolectados en áreas contaminadas de la región de Polonia, señalando valores muy superiores a los registrados por el presente trabajo.

El género más concentrador de $\mathrm{Pb}$ fue Chandrosterum $(4,13 \mathrm{mg} / \mathrm{kg})$; otros estudios en otros géneros mostraron concentraciones similares a los de Durkan et al. (2010), con concentraciones entre $1,38 \mathrm{mg} / \mathrm{kg}-4,79 \mathrm{mg} / \mathrm{kg}$, similares a esta investigación. Del mismo modo, Chaidez et al. (2015), en áreas marginadas de la zona sierra del estado de Durango, en México, reportaron en su estudio al hongo de la especie Pleorotus ostreatus concentraciones entre 0,59 - 0,99mg/ $\mathrm{kg}$, encontrándose los dos estudios dentro del rango de concentración de este hongo $(0,714 \mathrm{mg} /$ kg), colectado en Bahía Cispatá.

El género que presentó la máxima concentración de Cd fue Chandrosterum $(0,11 \mathrm{mg} / \mathrm{kg})$, siendo baja, de acuerdo con los resultados señalados por Durkan et al. (2010), que fueron de $0,511 \mathrm{mg} / \mathrm{kg}-2,044 \mathrm{mg} / \mathrm{kg}$ y similares a los de Chaidez et al. (2015), de $0,04-0,18 \mathrm{mg} / \mathrm{kg}$.

En el dendrograma de la figura 2, se muestran las agrupaciones y las asociaciones de los géneros de macromicetos con respecto a los metales pesados; se distinguen tres grupos definidos: uno, conformado por los géneros Pleorotus, Postia, Marasmiellus, Trametes, Daedelae, Ceriporia, Tyromices, Cerrena, Daldinia y Hexagonia; otro, por los géneros Crepidotus, Oudemansiella, Auricularia, Chandrosterum, Perenniporia, Polyporus, Earliella y Lenzites, indicando similitudes de comportamiento al concentrar metales pesados y, el último, donde se encuentra Mycena, con una tendencia diferente a los otros dos grupos, demasiado alejado.

En cuanto a los metales pesados, se conformaron tres grupos, uno, integrado por el $\mathrm{Pb}, \mathrm{Hg}, \mathrm{Cd}, \mathrm{Cu}, \mathrm{Cr}, \mathrm{Ni}$ y $\mathrm{Co}$ y estos, a la vez, forman otros subgrupos; otro, conformado por el $\mathrm{Zn}$ y, el último, por el Mn. El primer grupo son similares, posiblemente, en cuanto comportamiento de metales pesados concentrados por esos hongos. El Mn y el Zn mostraron comportamientos diferentes de forma independientes, de la que se destaca el Mn, que está más alejado del resto de hongos, debido a que este metal es muy abundante en la zona de forma natural y muestra más géneros que lo concentran, como indica la figura 3.

En el proceso de agrupamiento de los clusters o dendrograma, el género Mycena muestra un comportamiento particular lo que puede estar relacionado con la frecuencia (1) de colecta y las altas concentraciones de metales, como $\mathrm{Mn}, \mathrm{Cu}, \mathrm{Zn}, \mathrm{Ni}$ y Cd, esto muestra su alta capacidad de concentración, corroborado por Alonso et al. (2004). Por su parte, el Mn se mostró en un grupo muy diferente y aislado del árbol, debido a que ha sido el metal que se ha encontrado en mayor concentración en el medio y mayormente concentrado en los géneros de hongos. El Mn, se ha correlacionado con el segundo grupo, integrado por el $\mathrm{Zn}$, que fue concentrado altamente por los hongos y fue determinada su alta concentración en el ecosistema de Bahía Cispatá, en el estudio de Burgos-Núñez et al. (2017), se nota que este metal es ampliamente permitido en actividades agrícolas. Para la conformación del tercer conglomerado, que forma otros subgrupos, se aprecia, en primer lugar, que el Cd$\mathrm{Hg}$, formando el primer par de similitud, que luego se asocia con el $\mathrm{Pb}$, porque son elementos concentrados en pequeñas cantidades que no son esenciales y se encuentran en el medio por actividades antropogénicas (Burgos et al. 2014; Burgos-Núñez et al. 2017). Por otro lado, en este tercer grupo, se forma el siguiente par Ni-Co, que se asocia con el $\mathrm{Cu}$, demostrando la correlación de tres metales $\mathrm{Co}-$ $\mathrm{Ni}-\mathrm{Cu}$, donde usualmente el Co se encuentra en asociación con el $\mathrm{Ni}$ y estos dos con el $\mathrm{Cu}$, ya que poseen afinidades y concordancias de las constantes eléctricas (López \& Echeveste, 2012); por lo tanto, el $\mathrm{Hg}-\mathrm{Cd}-\mathrm{Pb}$, se asocia con Ni-Co-Cu y ambos lo hacen con el Cr. Luego, este conglomerado se asocia al segundo grupo al Zn y, finalmente, ambos se asocian al Mn.

La movilidad de metales pesados en el ecosistema manglar es debida a procesos naturales, como coagulación, floculación y precipitación del agua del cuerpo estuarino, a los cambios de $\mathrm{pH}$, temperatura y salinidad del agua, que se dan durante la mezcla que genera la remoción de estos elementos y a procesos antrópicos, como la construcción de diques, las labores agrícola-ganaderas que utilizan pesticidas, fertilizantes, la industria petrolera y las descargas de aguas residuales, que pasan al sustrato hospedero y terminan concentrándose en los hongos macromicetos de la Bahía Cispatá.

Si bien no se han encontrado concentraciones de metales pesados en cantidades alarmantes, se recomienda a las autoridades ambientales arbitrar las medidas preventivas, para erradicar o minimizar las causas de contaminación, por medio del control de las fuentes antropogénicas que liberan cantidades de metales pesados, tales como las actividades de producción y de la agricultura, la ganadería y otros sistemas industriales, que se llevan a cabo en las riberas del río Sinú y los caños y ciénagas que desembocan en la Bahía Cispatá y a las autoridades académicas y del área de la salud, tomar este estudio como señal de alerta para promover la evaluación del estado ecológico de la bahía.

Agradecimientos: Al Grupo de Investigación de Toxicología y Gestión Ambiental y al personal de los Laboratorios de Toxicología y Gestión Ambiental y de Ecología de la Universidad de Córdoba. Conflicto de intereses: El manuscrito fue preparado y revisado exclusivamente por los autores, quienes declaran no tener conflicto de interés que ponga en riesgo la validez de los resultados aquí presentados. Financiación: Los resultados expuestos en el manuscrito 


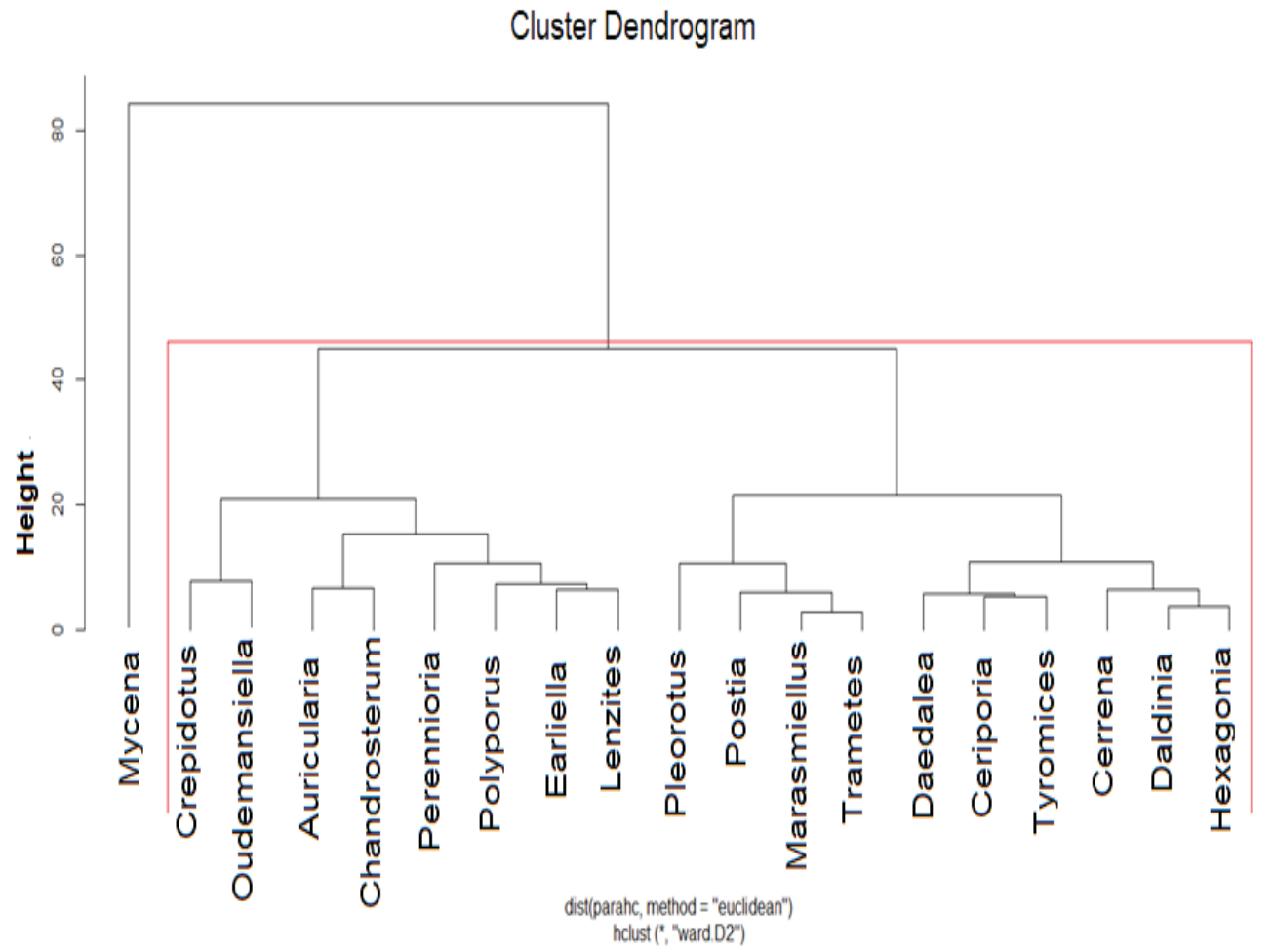

Figura 2. Dendograma comportamiento de géneros de hongos macromicetos en la Bahía de Cispatá.

\section{Cluster Dendrogram}

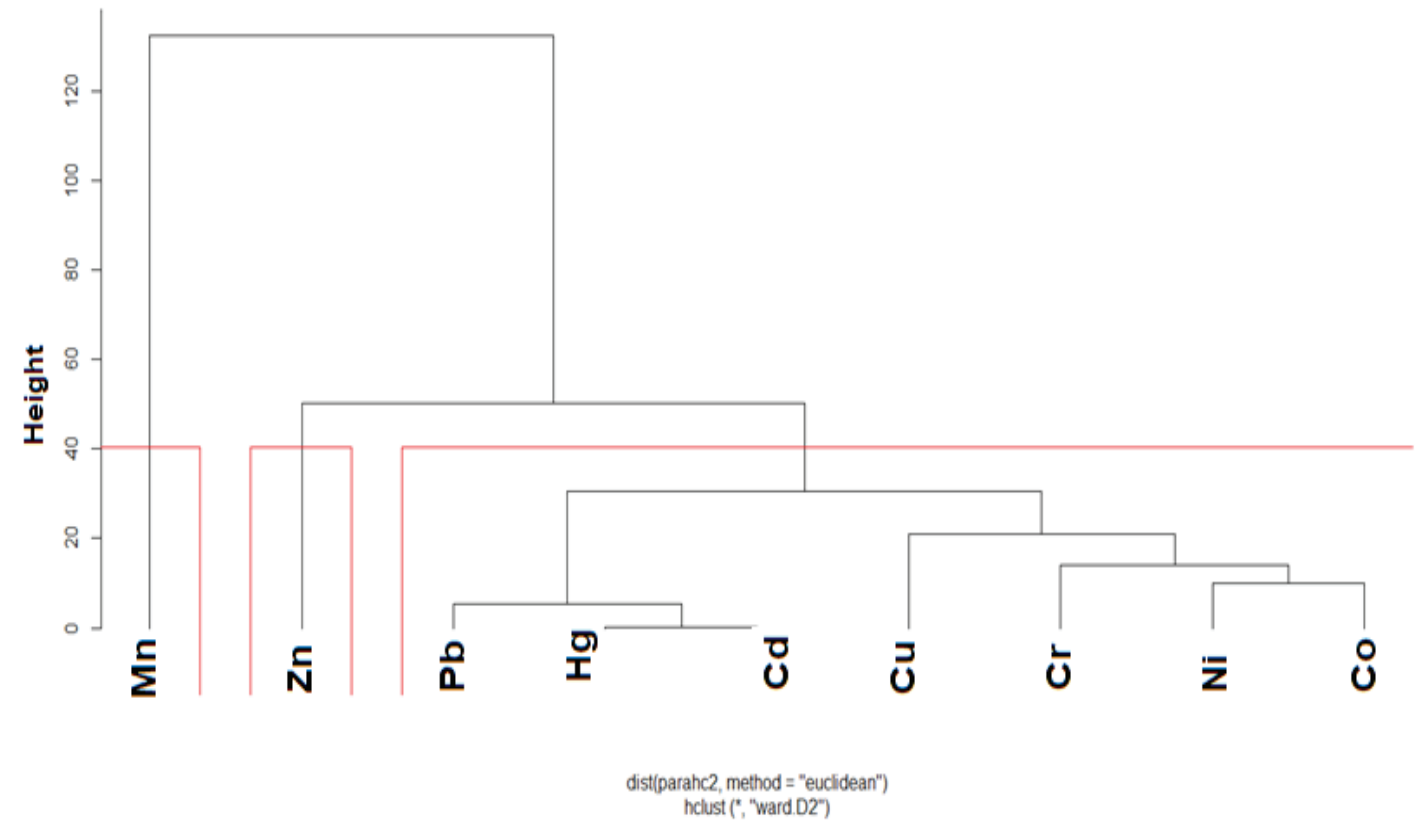

Figura 3. Dendograma comportamiento de la relación $\mathrm{Mn}, \mathrm{Cr}, \mathrm{Ni}, \mathrm{Cu}, \mathrm{Zn}, \mathrm{Co}, \mathrm{Hg}, \mathrm{Pb}$ y Cd en hongos macromicetos, en la Bahía de Cispatá-Colombia. 
son producto de un trabajo de investigación y los recursos de equipos, financieros y personales empleados son propio de los autores.

\section{REFERENCIAS}

1. ALONSO, J.M.; GARCÍA, M.; PÉREZ-LÓPEZ, M.; MELGAR, M.J. 2004. Acumulación de metales pesados en macromicetos comestibles y factores que influyen en su captación. Rev. Toxicología (Pamplona- España). 21(1):1115.

2. ARGOTA, G.; IANNACONE, J.; FIRMIA, R. 2013. Características de Gambusia punctata (PoeciliIdae) para su selección como biomonitor en ecotoxicología acuática en Cuba. The Biologist (Lima). 11(2):229-236.

3. ARTEAGA, G.; MARRUGO, J.Y.; SANCHEZ, J. 2017. Plaguicidas en canales de riego del distrito de La Doctrina. (Córdoba-Colombia). Temas Agrarios. 23(1):20-36. https://doi.org/10.21897/rta.v23i1.1143

4. BOROVIČKA, J.; ŘANDA, Z. 2007. Distribution of iron, cobalt, zinc and selenium in macrofungi. Mycol Progress (Czech Republic). 6(4):249-259. https://doi.org/10.1007/ s11557-007-0544-y

5. BURGOS, S.; MARRUGO, J.; URANGO, I.; NAVARRO, A. 2014. Mercury in Pelecanus occidentalis of the Cispatá bay (Colombia). Revista MVZ Córdoba. 19(2):4168-4174. https://doi.org/10.21897/rmvz.110

6. BURGOS-NÚÑEZ, S.; NAVARRO-FRÓMETA, A.; MARRUGO-NEGRETE, J.; ENAMORADO-MONTES, G.; URANGO-CÁRDENAS, I. 2017. Polycyclic aromatic hydrocarbons and heavy metals in the Cispatá Bay, Colombia: A marine tropical ecosystem. Marine Pollution Bulletin. 120:379-386.https://dx.doi.org/10.1016/j. marpolbul.2017.05.016

7. CARLILE, M.; WATKINSON, S.; GOODAY, G. 2001.The fungi. Second ed. London: Academic Press. 588p.

8. CHAIDEZ, A.; NARANJO, N.; DELGADO, E.; TORRES, R.; MEDINA, J.; MONREAL, H. 2015. Análisis toxicológico de seis especies de hongos silvestres comestibles de la región del Salto, Pueblo Nuevo Durango (México). Vidsupra visión científica. 7(1):6-10.

9. CHAN-CUPUL, W.; HEREDIA-ABARCA, G.; RODRÍGUEZVÁZQUEZ, R. 2016. Aislamiento y evaluación de la actividad enzimática ligninolítica de macromicetos del estado de Veracruz, México. Rev. Int. Contam. Ambie. 32(3):339351. https://doi.org/10.20937/RICA.2016.32.03.08
10. DEMIRBAS, A. 2001. Concentration of 21 metals in 18 species of mushrooms growing in the East Black Sea Region. Food Chemistry, (Turkey). 75(4):453-457. https://doi. org/10.1016/S0308-8146(01)00236-9

11. DUEÑAS, P.; CAMPOS, H.; QUIRÓS, A. 2012. Los crustáceos decápodos del Departamento de Córdoba, Colombia. Biodiversidad de la fauna de crustáceos decápodos en el Caribe colombiano. AV Akademikerverlag GmbH \& Co. KG Saarbrücken, Alemania. Editor: Editorial Académica Española. 258p.

12. DURKAN, N.; YUNUS, D.; UNVER, M.; ISILOGLU, M.; KABAR, K. 2010. Levels of trace metals in some macrofungi from Buyuk Menderes river basin, Turkey. Natura Montenegrina. Podgorica. 9(3):753-759.

13. FERIA, J.; GONZÁLEZ, H.; MARRUGO, J. 2010. Heavy metals in Sinú river, department of Córdoba, Colombia. Rev. Facultad de Ingeniería Universidad de Antioquia. $55: 35-44$

14. FRANCO, A.; URIBE, E. 2000. Hongos Agaricales y Boletales de Colombia. Biota Colombiana. 1(1):25-43.

15. GONZÁLEZ, E.; RAMÍREZ, M.; RUIZ, L. 2005. Diversidad biológica en Chiapas, Colegio de la Frontera Sur ECOSUR, Chiapas. p.71-89.

16. HERRERA, J.; RODRÍGUEZ, J.; COTO, J.; SALGADO, V.; BORBÓN, H. 2012. Evaluación de metales pesados en los sedimentos superficiales del río Pirro. Tecnología en Marcha (Costa Rica). 26(1):27-36. https://doi.org/10.18845/ tm.v26i1.1119

17. JOSELEAU, J.P.; RUEL, K. 1994. Wood polysaccharides and their degradation by fungi. En: Petrini,O.; Quellette, G.B. (eds). Host wall alterations by parasitic fungi. Editorial: St. Paul, Minn.: American Phytopathological Society. p.334387.

18. LEONOWICZ, A.; MATUSZEWSKA, A.; LUTEREK, J.; ZIEGENHAGEN, D.; WOJTACE-WASILEWSKA, M.; CHO, N.; HOFRICHTER, M.; ROGALSKI, J. 1999. Biodegradation of lignin by White rot fungi,Fungal Genetics and Biology. 27:175-185.

19. LODGE, D.J.; AMMIRAT, J.F.; O’DELL, T.E.; MUELLER, G.M.; HUHNDORF, S.M.; WANG, C.; STOKLAND, J.; SCHMIT, J.P.; RYVARDEN, L.; LEACOCK, P.; MATA, M.; UMAÑA, L.; WU, Q.; CZEDERPILTZ, D.L. 2004. Terrestrial and lignicolous macrofungi. In: Mueller, G.M.; Bills, G.F.; Foster, M.S. (eds). Biodiversity of fungi: inventory and monitoring methods. Elsevier Inc., San Diego. p.127-172. https://doi.org/10.1016/B978012509551-8/50011-8 
20. LÓPEZ, L.; ECHEVESTE, H. 2012. Paragénesis mineral del depósito tipo five element purísima-rumicruz, Jujuy. Rev. de la Asociación Geológica Argentina. 69(4):537-543.

21. LORENZO-MÁRQUEZ, H.; TORRES-DOSAL, A.; BARBA-MACÍAS, E.; ILIZALITURRI-HERNÁNDEZ, C.; MARTÍNEZ-SALINAS, R.; MORALES-LÓPEZ, J.; SÁNCHEZ-MORENO, I. 2015. Estimación de riesgo de exposición a metales pesados por consumo de plecos (Pterygoplichthys spp.) en infantes de comunidades ribereñas de los ríos Grijalva y Usumacinta, México. Rev. Internal Contaminación Ambiental. 32(2):153-164. http://dx.doi. org/10.20937/RICA.2016.32.02.02

22. MACÍAS, F.; CALVO DE ANTA, R. 2008. Niveles genéricos de referencia de metales pesados y otros elementos traza en los suelos de Galicia. Consejería de medio ambiente y desarrollo sostenible. 1:39-40.

23. MADERO, A.; MARRUGO, J. 2011. Detection of heavy metals in cattle, in the valleys of the Sinu and San Jorge rivers, department of Cordoba, Colombia. Rev. MVZ Córdoba, 16(1):2391-2401.

24. MARrugO, J.; PINEDO, J.; DIEZ, S. 2017. Assessment of heavy metal pollution, spatial distribution and origin in agricultural soils along the Sinú River Basin, Colombia. Environmental Research 154:380-388. https://doi. org/10.1016/j.envres.2017.01.02

25. MATA, M.; HALling, R.; MUEllER, G.M. 2003. Macrohongos de Costa Rica. Instituto Nacional de Biodiversidad (INBio). 240p.

26. MATA, M.; UMAÑA, L.; CHAVES, J. 2009. Documento borrador de referencia protocolo para la recolecta, descripción, identificación y mantenimiento de hongos. Instituto Nacional de Biodiversidad (INBio). (Costa Rica). $29 \mathrm{p}$.

27. MENDIL, D.; ULÜOZLÜ, Ö.D.; TÜZEN, M.; HASDEMIR, E.; SARI, H. 2005. Trace metal levels in mushroom samples from Ordu, Turkey. Food Chemistry. 91:463-467. https:// do.org/10.1016/j.foodchem.2004.06.028

28. MILLER, J.N.; MILLER, J.C. 2002. Estadistica y Quimiometría para Química Analítica, London: Prentice Hall. 286p.
29. MOYANO, A.; GARCÍA-SÁNCHEZ, A.; FERNÁNDEZTOIRÁN, L.M.; CHARRO, E. 2010. Metales pesados en hongos de áreas contaminadas. Rev. Ciências Agrárias (Portugal). 33(1):13-21.

30. ORTIZ-MORENO, M. 2010. Macromicetos en Zona Rural de Villavicencio. Revista Orinoquia. 14(2):125-132. https:/ / doi.org/10.22579/issn.2011-2629

31. RIVERO, A.; NIELL, S.; HEINZEN, H.; CESIO, M.; CERDEIRAS, M.; SOUBES, M. 2016. Selección de basidiomicetes nativos con capacidad de degradar xenobióticos usando el endosulfán como modelo. Rev. Laboratorio Tecnológico del Uruguay. 12:27-33.

32. RZYMSKI, P.; MLECZEK, M.; SIWULSKI, M.; GAૃSECKA, M.; NIEDZIELSKI, P. 2016. The risk of high mercury accumulation in edible mushrooms cultivated on contaminated substrates. J. Food Composition and Analysis. 54(8):2387-2393. https://doi.org/10.1016/j. jfca.2016.06.009

33. SÁNCHEZ, G. 2010. Centro de Memoria Histórica: La tierra en disputa Memorias de despojo y resistencia campesina en la costa Caribe colombiana (1960-2010). Ediciones Semana. 1: $224-476$.

34. SÁNCHEZ, H.; ULLOA, G.; TAVERA, H. 2004. Manual sobre zonificación y planificación para el manejo sostenible de los manglares por comunidades locales del caribe de Colombia. Ministerio de Medio Ambiente, Vivienda y Desarrollo Territorial - CONIFY - OIMT.

35. SOSA RODRÍGUEZ, T.; SÁNCHEZ NIEVES, J.; MELGAREJO, L. 2009. Papel funcional de los hongos en ecosistemas de manglar. Boletín de Investigaciones Marinas y Costeras - INVEMAR- (Colombia). 38(1):39-57. https://doi.org/10.25268/bimc.invemar.2009.38.1.161

36. SVOBODA, L.; ZIMMERMANNOVÁ, K.; KALAC, P. 2000. Concentrations of mercury, cadmium, lead and copper in fruiting bodies of edible mushrooms in an emission area of a copper smelter and a mercury smelter. Science of The Total Environment. 246:61-67. https://doi.org/10.1016/ S0048-9697(99)00411-8 